\title{
MS44-P03 | STRUCTURAL CHARACTERIZATION OF ZNO/GRAPHENE NANOCOMPOSITE
}

Sbarcea, Beatrice Gabriela (INCDIE ICPE-CA, Bucharest, ROU); Banciu, Cristina (INCDIE ICPE-CA, Bucharest, ROU); Marinescu, Virgil (INCDIE ICPE-CA, Bucharest, ROU); Patroi, Delia (INCDIE ICPE-CA, Bucharest, ROU); Lungulescu, Marius (INCDIE ICPE-CA, Bucharest, ROU)

Zinc Oxide $(\mathrm{ZnO})$ is a multifunctional semiconductor material and its used in applications involving sensors, lightemitting diodes (LEDs), piezoelectric devices, solar cells. Graphene, exhibits properties such as ultrahigh electron mobility, large surface area, high chemical and thermal stability, excellent electrical and optical properties.

The goal of this study is to obtain the porous 3D graphene network on nickel foam template by chemical vapor deposition (CVD) at high temperatures using methane as carbon source. ZnO-graphene (ZG) composite was developed using hydrothermal method to deposit zinc oxide directly on the 3D graphene network.

The prepared ZG were structurally characterized by X-ray diffraction, Raman technique and Scanning Electron Microscopy. The analysis of X-ray diffraction showed that the samples had hexagonal wurtzite structure. From SEM imagine we obtain hexagonal ZnO nanorods structures.

ZnO-graphene nanocomposites were synthesized in order to develop a good material for energy applications. 